\title{
COMBATER O ESTIGMA ASSOCIADO AO SOFRIMENTO PSÍQUICO: UM RELATO DE EXPERIÊNCIA DA EDUCAÇÃO PROFISSIONAL EM SAÚDE
}

\author{
COMBATIR EL ESTIGMA ASOCIADO CON EL TRASTORNO MENTAL: UNA \\ DESCRIPCIÓN DE LA EXPERIENCIA DE LA EDUCACIÓN PROFESIONAL EN \\ SALUD
}

\begin{abstract}
FIGHTING STIGMA ASSOCIATED WITH MENTAL DISORDER: AN ACCOUNT OF THE EXPERIENCE OF PROFESSIONAL HEALTH EDUCATION
\end{abstract}

\author{
Marcela dos SANTOS FERREIRA ${ }^{1}$
}

RESUMO: As atitudes estigmatizantes, associadas aos indivíduos em sofrimento psíquico, presentes em estudantes e trabalhadores da área da saúde são reproduzidos no cuidado com a saúde, produzindo adversidades, além daquelas já geradas pelo próprio sofrimento psíquico. Uma forma de combater esse problema social é o investimento da educação profissional em um ensino antiestigmatizante. $\mathrm{O}$ objetivo deste artigo é apresentar um relato de experiência de uma intervenção na disciplina de saúde mental, do curso técnico em enfermagem, constituída pelo planejamento e implementação de uma prática de ensino, voltada para o enfrentamento do estigma. Esta prática mostrou redução do estigma entre alunos técnicos de enfermagem e a mesma esteve relacionada a uma organização da disciplina, com a inclusão de estratégias educacionais que defendam o modelo de atenção psicossocial e que promovam o contato dos estudantes com casos de indivíduos em sofrimento psíquico.

PALAVRAS-CHAVE: Estigma social. Transtornos mentais. Educação. Educação em enfermagem.

RESUMEN: Las Actitudes Estigmatizantes asociadas con personas con enfermedades mentales, presentes en estudiantes y profesionales de la salud, se reproducen en la atención salud, causando adversidades, además de las ya generadas por enfermedades mentales. Una forma de combatir este problema social es invertir en educación profesional contra el estigma. El propósito de este artículo es presentar un informe de experiencia de una intervención en la disciplina de salud mental, del curso técnico en enfermería, que consiste en planificar e implementar una práctica docente, con el objetivo de abordar el estigma. Esta práctica mostró una reducción en el estigma entre los estudiantes técnicos de enfermería y estuvo relacionada con la organización de la disciplina, con la inclusión de estrategias educativas que defienden el modelo de atención psicosocial y que promueven el contacto de los estudiantes con casos de personas con enfermedades mentales.

PALABRAS CLAVE: Estigma social. Transtornos mentales. Educación. Educación em enfermeria.

${ }^{1}$ Centro Federal de Educação Tecnológica Celso Suckow da Fonseca (CEFET), Nova Iguaçu - RJ - Brasil. Docente da Coordenação de Enfermagem. Mestre em Educação Profissional em Saúde (FIOCRUZ/RJ). ORCID: http://orcid.org/0000-0001-7831-1245. E-mail: cceccella@hotmail.om 
ABSTRACT: The stigmatizing attitudes, associated with individuals with mental illness, present in students and health workers are reproduced in health care, producing adversities, in addition to those already generated by the mental illness itself. One way to combat this social problem is to invest in professional education in anti-stigmatizing education. The objective of this article is to present an experience report of an intervention in the mental health discipline, of the technical course in nursing, consisting of the planning and implementation of a teaching practice, aimed at facing the stigma. This practice showed a reduction in stigma among nursing technical students and it was related to an organization of the discipline, with the inclusion of educational strategies that defend the psychosocial care model and that promote students' contact with cases of individuals with mental illness.

KEYWORDS: Social stigma. Mental disorders. Education. Education. Nursing.

\section{Introdução}

O enfrentamento do estigma vem sendo considerado por estudiosos e instituições voltadas para o campo da saúde uma temática importante para a melhoria da promoção da saúde a pessoas com algum tipo de sofrimento psíquico. Essa preocupação demonstra que existe a necessidade de derrubar essa barreira real, de forma que facilite a prestação de cuidados a essa parcela da população. A Organização Mundial da Saúde (OMS) em seu Relatório Mundial da Saúde, cujo foco são as novas concepções de saúde mental, aborda essa problemática indicando que “[...] políticas são necessárias para assegurar o fim do estigma e da discriminação bem como a implantação da prevenção e do tratamento eficaz, com financiamento adequado" (OMS, 2001, p. 13). A partir dessa perspectiva, caminhos devem ser traçados para que esse objetivo possa ser alcançado.

O entendimento do estigma presente neste estudo perpassa a publicação do livro "Identidade deteriorada", do sociólogo Erwin Goffman. Para o autor o estigma surge da relação entre pessoas normais e estigmatizadas, consideradas como anormais e desviantes pela sociedade. $O$ estigma, então, seria uma preconcepção elaborada pelos normais a partir de atributos considerados comuns e naturais que é transformada em expectativa normativa, usada de modo rigoroso (GOFFMAN, 1988).

O estigma pode se apresentar de diversas formas, sendo o principal tipo o social, pois é partir dele que todos os outros se formam (autoestigma, profissional, familiar e estrutural). Esse nível de estigma faz parte da estrutura da sociedade e cumpre uma função, bem descrita por Ahmedani (2011, p. 4) "É uma crença sustentada por uma grande parcela da sociedade em que as pessoas com a condição estigmatizada são menos iguais ou fazem parte de um grupo inferior. Nesse contexto, o estigma é incorporado no âmbito social, para criar inferioridade". Desta forma acaba por produzir dificuldades além daquelas já intrínsecas ao sofrimento psíquico. 
Sabe-se que as atitudes estigmatizantes, segundo Cavazza (2005), são produtos sociais e, portanto, seu enfrentamento em primeiro lugar deve ser mediante processos que se colocam nas relações entre os grupos sociais. Todavia, não se pode esquecer que mudanças intraindividuais também permitem transformações das atitudes. Para que elas ocorram a estratégia que, segundo Corrigan et al., (2001), apresenta melhores resultados é a associação entre educação e contato, em especial com grupos de pessoas que possuem algum tipo de relação com os portadores de transtornos mentais.

A educação é considerada, por Arboleda-Floréz e Stuart (2012), uma das abordagens para a redução do estigma e, que ao ser aplicada à população, seja composta por leigos, portadores de transtornos mentais, estudantes ou profissionais de saúde, tem como propósito "substituir mitos e a desinformação com concepções precisas sobre a natureza e a prevalência de transtornos mentais, melhorando assim a carga de conhecimento global em saúde mental" (ARBOLEDA-FLÓREZ; STUART, 2012, p. 461).

Diante desse panorama, algumas questões surgiram para nortear esta experiência com alunos técnicos em enfermagem. Uma indagação importante é se existe influência do ensino de saúde mental no enfrentamento do estigma associado ao transtorno mental em alunos técnicos de enfermagem. Esse questionamento surgiu a partir da observação de estudos sobre estigma em profissionais de saúde, mesmo eles apresentando conhecimento de tais transtornos. De acordo com Nordt et al., (2006) profissionais de saúde mental não diferem do público em geral sobre a sua desejada distância social de indivíduos com algum transtorno mental. A simples existência de uma disciplina ou conteúdo voltado para o assunto, durante a formação profissional, não parece ser satisfatória para minimizar o problema.

Essas dúvidas levaram a outro tipo de questionamento, agora relativo não à existência de um conteúdo formativo acerca de transtornos mentais, mas sim, como o ensino de saúde mental produz mudanças no nível de estigma associado ao transtorno mental, em alunos técnicos de enfermagem.

Perante essas indagações houve a necessidade de se aplicar uma intervenção no ensino de saúde mental, em uma perspectiva antiestigmatizante, a alunos técnicos de enfermagem. O relato desta experiência é o objetivo deste artigo, apresentando a prática e vivência como docente da disciplina.

\section{Metodologia}

O cenário de estudo foi a disciplina de saúde mental na qual exerço a função de docente, ofertada a alunos do curso médio técnico de enfermagem no ano de 2019, apresentando carga horária 
de 108 horas, da Unidade Descentralizada do Centro Federal de Educação Tecnológica Celso Suckow da Fonseca (CEFET/Uned-NI) situada no município de Nova Iguaçu (RJ), que oferece o Curso Médio Técnico de Enfermagem.

A disciplina de saúde mental foi construída a partir de uma concepção antiestigmatizante, visando a promover uma mudança nas atitudes estigmatizantes dos alunos em relação aos indivíduos em sofrimento psíquico. Para tanto, a disciplina foi concebida na perspectiva do conhecimento combinado com uma reflexão crítica e problematizadora, em torno do sofrimento psíquico.

Assim, a proposta da disciplina de saúde mental esteve em consonância com Bezerra Junior (2007), quando diz que se deve provocar no aluno capacidade crítica e competência técnica para se envolver numa prática de cuidado que se constitua num exercício de transformação para todos os envolvidos: pacientes, profissionais e a comunidade. Isso permite uma possível construção de uma nova atitude em relação ao estigma e seus problemas associados.

A disciplina contou com o auxílio fundamental de instrumentos pedagógicos estratégicos que aumentaram o contato com histórias de indivíduos em sofrimento psíquico, podendo citar vídeos, filmes, reportagens jornalísticas, livros, documentários, artigos científicos, entre outros, que são descritos durante o relato de experiência.

Este relato de experiência foi construído de forma que fossem expressos experiências, acontecimentos e comportamentos individuais e coletivos dos alunos e que pudessem comunicar o estigma associado ao transtorno mental em estudantes técnicos em enfermagem e sua relação com o ensino de saúde mental em uma perspectiva antiestigmatizante.

\section{Resultados e discussão}

O primeiro contato com os alunos ocorreu em sala de aula onde foi apresentada a disciplina saúde mental, com seus objetivos e propostas de atividades educacionais. Nesse momento inicial de encontro, uma simples enquete foi feita para descobrir um pouco do conhecimento prévio dos alunos, que constava da pergunta aberta: Qual o local de atendimento para uma pessoa em sofrimento psíquico? Nesse instante foi usada também outra palavra de mais fácil compreensão por eles, como doente mental. As respostas em ordem de frequência foram: Hospital psiquiátrico (grande maioria); clínica psiquiátrica; manicômio e consultório de psiquiatria.

As respostas demonstraram que existe um predomínio da noção institucionalizante da assistência ao indivíduo em sofrimento psíquico pelos alunos e que a disciplina de saúde mental teria que usar essa informação como parâmetro para estratégias que transformassem essa noção tão relacionada a crenças e atitudes estigmatizantes. 
No intuito de que os alunos conseguissem compreender de forma mais efetiva alguns temas relacionados às aulas teóricas, em especial a temática institucionalização e tratamento asilar, foi proposta uma atividade extra à sala de aula que consistia na visita virtual da exposição sobre o Hospício Pedro II do Centro Cultural da Saúde e na leitura de trechos do livro "Holocausto brasileiro" (Autoria de Daniela Arbex, 2013). Ainda, para fomentar uma discussão com os alunos acerca de o tratamento asilar, os alunos assistiram aos documentários "Em nome da razão" (direção de Helvécio Ratton, 1979) e "Holocausto Brasileiro" (direção de Daniela Arbex e Armando Mendez, 2016).

A partir desse conjunto de atividades, no debate com os alunos percebeu-se, que eles pouco conheciam da história dos hospitais psiquiátricos apresentados pelos vídeos, como também a forma não humana de assistência a essas pessoas.

Com o propósito de apresentar a realidade do tratamento asilar na psiquiatria, isso fez com que os alunos reconhecessem que os comportamentos estereotipados que os as pessoas apresentavam nos documentários não era essencialmente produto de seu sofrimento psíquico, mas em grande parte relacionado com o isolamento social e com o tratamento subumano que recebiam. Algumas dessas características são citadas pelos alunos, como retraimento, fala empobrecida e aparência descuidada.

Em especial a leitura do livro "Holocausto brasileiro", que estabelece um contato maior com histórias individuais do Manicômio de Barbacena, aumentou a percepção dos alunos de que a institucionalização não é o melhor mecanismo de assistência e que ele é responsável pela despersonalização, cronificação de alguns sofrimentos e piora de condições clínicas, sociais, familiares e psicológicas.

Como os documentários e o livro também descrevem algumas mudanças ocorridas na assistência a essas pessoas em sofrimento psíquico, os alunos já começaram a ter contato com outro modelo de atenção à saúde mental (que até então pouco conheciam), o que os levou a identificar que é possível mudar a realidade de pessoas que passaram até mesmo por longo tempo de internamento.

O propósito da disciplina de saúde mental em uma perspectiva antiestigmatizante foi discutir o estigma em todos os momentos possíveis, por isso, foi proposto que os alunos apontassem estigmas associados ao indivíduo em sofrimento psíquico. Eles conseguiram reconhecer a existência dos seguintes estigmas: irrecuperável; violentos; identificável pela aparência; fala e comportamento alterados; descuidados; não podem trabalhar ou estudar e possuem retardos.

Nesse contexto de descrição de estigmas encontrados na sociedade, levantou-se um debate de por que os alunos, ou a sociedade de modo geral, apresentavam essa imagem sobre pessoas com quem não tem contato direto. A mídia foi reconhecida pelos alunos como sendo um fator importante para a difusão de certas características que acabam sendo generalizadas a todos os indivíduos em sofrimento psíquico e em especial a televisão apresenta uma imagem distorcida destas pessoas que não são 
conhecidas por parte da sociedade, o que as fazem ainda terem estereótipos que estão ligados a um passado de tratamento manicomial.

Esta imagem apresentada pelos próprios alunos vai ao encontro do pensamento de Gil (2010, p. 100), que relata que
Apesar de ter aumentado o nível de informação e sensibilização da opinião pública ainda é notória a permanência de estereótipos negativos acerca das doenças e dos doentes mentais que, de certo modo são potenciados pela concentração da opinião pública em episódios negativos que tendem a acentuar os sentimentos de insegurança por parte da sociedade em geral, contribuindo para a criação de categorias e estereótipos que, por seu lado, estão na origem dos processos de categorização e de discriminação social.

De forma a dar continuidade à discussão dos estigmas, em especial aos associados à irrecuperabilidade, restrição social e violência, foi proposto assistir um filme que traz uma importante reflexão sobre a transformação de um hospital psiquiátrico: "Nise - O coração da loucura" (direção de Roberto Berlinder, 2015), mas com o olhar voltado para os estigmas já citados.

Após a exibição do filme, alguns alunos, baseados em alguns trechos do vídeo, disseram que algumas características violentas dos indivíduos em sofrimento psíquico poderiam estar relacionadas à forma como eles eram tratados no ambiente hospitalar, sendo a agressividade uma resposta a pessoas ou ambientes opressores, o que demonstrou que alguns estudantes começaram a dissociar a imagem da violência do sofrimento psíquico. No entanto, essa ainda não se mostrou ser uma opinião da maioria da turma.

O Movimento de Luta Antimanicomial foi apresentado como uma temática importante de ser conhecida, por apresentar ações significativas desse movimento no campo do estigma e mostrar que a luta contra a estigmatização não é localizada e sim uma ação de parte de trabalhadores e apoiadores da área da saúde mental e que foi por meio desse movimento que alguns estigmas foram postos em discussão e ganharam visibilidade na sociedade, sendo o mais discutido com os alunos a questão da restrição e isolamento social.

Dentro dessa temática, foram usadas para ampliar a discussão da luta antimanicomial algumas legislações antigas que versavam sobre a assistência psiquiátrica, demonstrando-se por meio delas como o indivíduo em sofrimento psíquico era visto pela sociedade, assim como a importância dos movimentos em prol da destituição do manicômio e suas ideias reproduzidas na sociedade.

O estudo associado de legislações e dos movimentos de luta antimanicomal suscitaram questionamentos relacionados à mudança assistencial aos indivíduos em sofrimento psíquico, há uma transformação no pensamento dos profissionais de saúde, e para tanto usaram como exemplo o filme 
"Nise - O coração da loucura", no qual pode ser visto que foram necessárias mudanças nas atitudes dos funcionários.

Como continuidade da aula sobre o movimento de luta antimanicomial, os alunos tiveram uma sequência de aulas voltadas para a discussão da Reforma Psiquiátrica, legislações associadas a ela e as peculiaridades do modelo de atenção à saúde mental proposto pela reforma.

Em se tratando da discussão sobre modelos alternativos ao manicômio, a dúvida eminente do grupo estava associada em como tratá-los fora de uma internação e se era possível. A presença de alguns estigmas pareceu estar associada com o descrédito na desinstitucionalização, pois a justificativa dos alunos para não acreditarem nessa possibilidade está agregada à crença de que os portadores de transtorno mental teriam dificuldade de viver fora de o ambiente hospitalar, por conta da dependência que teriam desse tipo de ambiente, assim como pelo risco que poderiam trazer à sociedade, baseados na ideia de que são pessoas agressivas e instáveis.

O entendimento por parte dos alunos sobre o funcionamento e a eficácia das estruturas que favorecem o processo de desinstitucionalização (Centro de atenção psicossocial - CAPS e residência terapêutica) ainda era difícil e pôde ser justificado pelo pouco conhecimento prático sobre esses locais, além de existirem algumas questões que se tornaram barreiras para o aluno acreditar na possibilidade de um tratamento comunitário, como o descrédito na independência das pessoas em sofrimento psíquico e suas capacidades de convívio em grupo ou numa casa dividida por pessoas que apresentam os mesmos tipos de problemas.

De forma a ampliar o conhecimento dos alunos sobre o CAPS e na tentativa de diminuir seu descrédito sobre essa instituição, considerada peça central para a continuidade do processo da reforma psiquiátrica, os alunos tiveram a oportunidade de assistir a três reportagens jornalísticas veiculadas na televisão denominadas "Saúde Mental - CAPS” (Canal Bandeirantes, 2013), "Saúde Mental Residências Terapêuticas" (Canal Bandeirantes, 2012), "Quebrando Paradigmas" (Canal Bandeirantes, 2013), que documentavam o funcionamento de um CAPS e outras estruturas associadas a ela como cooperativas sociais e a residência terapêutica.

A percepção que apresentaram após assistirem aos vídeos foi de que as pessoas frequentadoras do CAPS, assim como os funcionários, têm um comportamento distinto daqueles que estão em hospitais psiquiátricos. A diferença de ambiente e do cuidado prestado estaria relacionada, segundo os alunos, a uma melhor reabilitação de tal forma que passa a ser possível acreditar que essas pessoas podem viver fora de um ambiente de isolamento.

Este contato indireto com indivíduos em sofrimento psíquico aliado à educação em saúde, principalmente a voltada para o atendimento extra-hospitalar com foco na desinstitucionalização, 
substitui mitos por realidade, afetando positivamente as atitudes em relação aos estigmas (FOSTER et al., 2010; ROBERTSON-HICKLING; PAISLEY, 2011; LÓPEZ et al., 2008).

Em meio às atividades educativas durante o ano letivo, os alunos participaram e construíram ações para o evento anual da instituição denominado Semana de Enfermagem, que nesse ano, coincidentemente, ocorreu no mesmo período em que se comemorou a Semana da Luta Antimanicomial. Aproveitando a justaposição das datas foi proposto aos alunos que desenvolvessem uma atividade em que fosse apresentada e difundida a ideia da luta antimanicomial aos participantes do evento.

Durante o mês que antecedeu o encontro os alunos preparam um workshop para divulgação do trabalho de criação de arte em camisas que remetia à luta antimanicomial e o estigma associado aos indivíduos em sofrimento psíquico.

A escolha das estampas que seriam impressas foi feita nas semanas que antecederam o evento, em debates que revelou o quanto cada aluno tinha apreendido dessa temática, como também foi percebido que o envolvimento dos alunos nessa atividade aumentou a participação nas aulas em que houve discussão em torno do estigma e da reforma psiquiátrica.

Como forma de discutir o estigma da responsabilização individual das pessoas pelos seus transtornos mentais, os alunos assistiram a um documentário intitulado "TOC - A história real de uma vida perdida em pensamentos" (realizado pelo canal Odisea, 2008), que ao mesmo tempo em que complementou a aula teórica sobre transtornos ansiosos também descrevia casos em que as pessoas com ansiedade e transtorno obsessivo-compulsivo (TOC) relatavam como eram julgadas por seus problemas de forma estigmatizadora.

Como consequência, os estudantes conseguiram identificar que as pessoas com transtornos ansiosos, por serem consideradas pela sociedade como conscientes de seus atos, acabam sendo repreendidas pela falta de força de vontade em melhorar seus comportamentos e que alguns portadores acabam por concordar com esse pensamento, o que fez os alunos se lembrarem da aula sobre autoestigma.

Já para discutir estigmas relacionados aos transtornos de humor, em especial a depressão, os alunos tiveram inicialmente um conteúdo teórico sobre os transtornos e outras três atividades: leitura prévia do artigo "Experiências do estigma na depressão: um estudo transcultural" (autoria Moreira e Telles, 2008), discussão de casos a partir de cartas de suicidas e uma palestra sobre medicalização dos sentimentos.

O conteúdo teórico proposto estava voltado principalmente para estimular o entendimento de um modelo causal multifatorial da depressão. No entanto, quando os alunos foram apresentados às medicações antidepressivas, notou-se que os mesmos atribuíram à bioquímica como sendo a causa 
mais importante, o que levou alguns alunos a entenderem que a reabilitação estaria associada apenas à medicação, o que tenderia a gerar responsabilizações individuais nas situações nas quais não há resposta medicamentosa.

No prosseguimento das discussões em torno da depressão, histórias de pessoas com depressão e que cometeram suicídio foram analisadas a partir de cartas escritas por elas. Essa análise de casos de portadores de depressão, mesmo sendo superficial, fez com que os alunos conseguissem identificar inúmeros fatores causais da depressão numa mesma pessoa. Ao mesmo tempo em que os estudantes conseguiram identificar vários fatores predisponentes para a depressão, também notaram que nas cartas os indivíduos tendiam a apontar uma única causa para os seus problemas.

As percepções dos alunos ao lerem as cartas foram muito diversas. Alguns entenderam de que a baixa da autoestima é uma manifestação da depressão, que, segundo eles, é piorada pela culpabilização individual por seu transtorno, enquanto outros sustentaram que os portadores de depressão internalizam com mais facilidade o estigma da culpa.

Ao começar as aulas sobre esquizofrenia, os alunos já haviam assistidos o documentário sobre funcionamento do CAPS e das cooperativas sociais (assistido no início da disciplina), e realizado o workshop sobre luta antimanicomial. Essas experiências concorreram para que os alunos fizessem críticas e questionamentos importantes quando assistiram vídeos jornalísticos recomendados para as aulas sobre esquizofrenia: "Seguir em frente - Vencendo a esquizofrenia” (canal Rede Vida, 2014); "A casa de saúde Dr. Eiras e reforma psiquiátrica" (Canal TV Brasil, 2015); "Eleanor Longden - As vozes da minha cabeça" (Site TED, 2013); "Eu não sou um mostro - Esquizofrenia" (Site TED, 2017); “Ouvidores de vozes" (canal Futura, 2017) e o documentário "Di capacitados" (Direção de Pepe Martinez, 2015).

A turma considerava no início do ano letivo a esquizofrenia como o principal exemplo de sofrimento psíquico psiquiátrico e suas manifestações se encaixavam no que eles consideravam o 'típico louco'. Em no decorrer do curso essa visão pode ser modificada.

Apesar da diminuição aparente da presença de alguns estigmas nos alunos, a associação da violência com a esquizofrenia ainda era muito arraigada na turma, e por isso se fez necessário manter um foco especial nessa questão durante as aulas de esquizofrenia.

Essas atitudes estigmatizantes se apresentavam bem consolidadas, a ponto de poderem ser caracterizadas, segundo o conceito de Cavazza (2005), como atitudes fortes, considerando as características de ser persistentes e resistentes às mudanças, como também causadoras de grande impacto nos processos de elaboração das informações e sobre o comportamento.

A grande dificuldade em trabalhar a questão da violência com os alunos se deu pelo fato de eles só tomarem conhecimento, dos casos de esquizofrenia associados com alguma manifestação de 
agressividade, fazendo-os crer que todos os portadores de esquizofrenia e outros transtornos seriam pessoas violentas, e com o avanço do curso conseguiram identificar que essas associações são devidas, em parte, pela população não aceitar que casos de violência extrema podiam ser provocados por pessoas consideradas normais.

De forma a melhorar o entendimento dos alunos sobre a associação de violência com a esquizofrenia ou transtornos que apresentem quadros psicóticos foi indicada a leitura do artigo "Esquizofrenia, psicopatologia e crime violento: uma revisão das evidências empíricas" (autoria Teixeira et al., 2007) e a visita aos sites Entendendo a Esquizofrenia (desenvolvido pelo psiquiatra Leonardo Palmeira) e Memórias de um Esquizofrênico (desenvolvido por Júlio Cezar Oliveira), que abordavam entre outros assuntos o real quadro da violência nessa população.

Após entrarem em contato com esses materiais, um assunto apontado por vários alunos foi o uso de drogas lícitas ou ilícitas como fator que antecedeu o comportamento violento das pessoas com esquizofrenia, o que levantou a discussão referente à droga como sendo um fator estimulante para qualquer ato de violência, já que para alguns alunos ela também estimula a violência em pessoas que não são diagnosticadas com algum transtorno mental.

Em relação às formas de tratamento da esquizofrenia, essas foram abordadas numa aula que também se preocupou em discutir os conceitos de cura, recuperação e reabilitação existentes na área da saúde. A intenção desse conteúdo era discutir estigmas como o de irrecuperabildade e incapacidade.

Essa aula em questão foi um momento em que os alunos se lembraram de inúmeros conteúdos já vistos na disciplina em que houve uma efetiva reabilitação de indivíduos em sofrimento psíquico, sendo os mais citados os casos em que as pessoas voltaram a ter relacionamentos amorosos e a trabalhar.

Partindo do conhecimento já estabelecido em literatura de que alguns estigmas estão relacionados com a maneira como as pessoas entendem as causa dos sofrimentos psíquicos, foi proposto aos alunos a assistir ao documentário "Estamira" (direção de Marcos Prado, 2006), e a partir dele apontarem possíveis atitudes estigmatizantes que teriam como origem o entendimento da causalidade da esquizofrenia. Os alunos ressaltaram que, no filme, familiares da personagem sugerem que o surgimento do transtorno deveu-se por uma punição religiosa e sinalizam outros estigmas associados à causalidade que foram observados, nas situações em que pessoas temiam que os filhos pudessem ter o transtorno, já que acreditavam na hereditariedade do problema.

Sabe-se que a forma como os transtornos mentais são explicados interferem na modulação das atitudes estigmatizantes e que o modelo psicossocial, aplicado na disciplina, é, segundo Luís (2011), a forma de explicar os transtornos mentais menos associada ao aumento da estigmatização ao 
contrário das explicações genéticas e biológicas que conforme Romano (2016), estão relacionadas a atitudes mais negativas em relação aos portadores de transtornos mentais.

De forma que o aluno pudesse visualizar como o estigma pode impregnar a atuação profissional, foi realizada uma discussão com eles a partir da leitura de artigos que versam sobre as concepções e percepções dos transtornos mentais por trabalhadores da saúde mental: (1) "A concepção de loucura e do seu tratamento entre os trabalhadores de saúde mental de uma instituição prestadora de serviço em nível secundário de atenção" (autoria de Pugin, Barbério e Filizola, 1997); (2) "Saúde mental na percepção de trabalhadores de um CAPS" (autoria de Caeran e Dias, 2015).

Os alunos extraíram trechos dos artigos que faziam referência a falas de trabalhadores que diferenciavam as pessoas com algum tipo de problema classificado como mais simples, das que realmente eram diagnosticadas com um transtorno mental. $\mathrm{O}$ que despertou o debate sobre o que levava o trabalhador da saúde a fazer essa diferenciação. As explicações também extraídas dos artigos seriam, segundo os alunos, a capacidade de viver sem internação e a presença de agressividade.

O que também foi lembrado era a concepção de alguns trabalhadores da área de saúde mental sobre as causas dos sofrimentos psíquicos, que em sua maioria os associavam a questões sociais, em especial à pobreza, e poucos trabalhadores indicaram o transtorno como um problema puramente biológico.

Alguns alunos concluíram que os indivíduos em sofrimento psíquico, ao saberem que trabalhadores da saúde possuem algum estigma sobre eles, poderiam não procurar sua assistência, como também indicaram que eles podem ser tratados de maneira incorreta ao não ser considerados realmente portadores de algum sofrimento psíquico, conforme relata o artigo lido. Já segundo outro grupo de alunos, estas pessoas poderiam ser mal atendidas pela presença do medo desses trabalhadores.

\section{Considerações finais}

As atitudes estigmatizantes, em relação ao transtorno mental, configuram-se como um fenômeno complexo que merece especial atenção da comunidade científica e acadêmica, tendo em vista que o ambiente acadêmico é essencial não só para desenvolvimento de conhecimentos e competências, mas, também, constitui um espaço para a promoção de atitudes antiestigmas.

Este relato de experiência apresentou a organização da disciplina Saúde Mental, com a inclusão de estratégias educacionais que promoveram o contato dos alunos com indivíduos em sofrimento psíquico e que defenderam o modelo de assistência psicossocial a estas pessoas. Estes 
recursos adotados durante as aulas parecem ter relação com a modificação de algumas atitudes estigmatizastes dos discentes.

Partindo da informação dos próprios alunos de que a mídia fortalece estigmas, ao veicular histórias que nem sempre são verdadeiras ou partindo de generalizações, o enfrentamento do estigma pela disciplina de saúde mental considerou o uso da mídia, mas num contexto de desestigmatização, usando vídeos em que histórias reais de portadores de transtornos mentais foram a base para que os alunos conseguissem ao longo da disciplina formar novos conhecimentos ou substituir crenças estigmatizantes.

A presença de atitudes estigmatizantes, que indicavam a noção de que todos os indivíduos com sofrimento mental são agressivos, foi a que esteve mais presente no decorrer da disciplina. Por isso, pode-se afirmar que a agressividade associada ao transtorno mental foi o exemplo de estigma cujo enfrentamento foi o mais moroso e complexo.

Após a intervenção antiestigma, ocorrida na disciplina de saúde mental, pôde-se inferir que existem alguns aspectos sobre o estigma que, quando discutidos em um curso da área da saúde, fomentam mudanças de atitudes. Mas, para isso, foi imprescindível o caráter não pontual das discussões que envolviam o tema. Os diversos assuntos e estratégias de ensino estavam sempre envolvidos com a discussão do estigma, fazendo com que os alunos ao longo da disciplina fossem expostos a informações e reflexões que os estimularam a desenvolver uma atitude menos estigmatizante.

Apesar da grande estabilidade que as atitudes apresentam, tornando-as difíceis de serem modificadas, foi possível produzir algumas transformações atitudinais nos alunos técnicos de enfermagem no que diz respeito ao estigma associado ao indivíduo em sofrimento psíquico, por meio do ensino de saúde mental.

\section{REFERÊNCIAS}

AHMEDANI, B. K. Mental health stigma: society, individuals, and the profession. J Soc Work Values Ethics, Washington, DC, v. 2, n. 8, p. 1-16, 2011. Disponível em: https://www.ncbi.nlm.nih.gov/pmc/articles/PMC3248273/. Acesso em: 05 nov. 2016.

ARBOLEDA-FLÓREZ, J.; STUART, H. From sin to science: fighting the stigmatization of mental illnesses. Revue Canadienne de Psychiatrie, Canadá, v. 57, n. 8, p. 457-463, 2012. Disponível em: http://connection.ebscohost.com /c/articles/78854969/from-sin-science-fighting-stigmatizationmental-illnesses. Acesso em: 09 ago. 2016.

BEZERRA JUNIOR, B. Desafios da reforma psiquiátrica no Brasil. Physis: Rev. Saúde Coletiva. Rio de Janeiro, v. 2, n. 17, p. 243-250, 2007. Disponível em: http://www.scielo.br/scielo.php? script=sci_arttext\&pid=S0103-73312007000200002. Acesso em: 05 nov. 2016. 
CAVAZZA, N. Psicologia das atitudes e das opiniões. São Paulo: Loyola, 2005.

CORRIGAN, P.W et al. Three strategies for changing attributions about severe mental illness. Schizophrenia Bulletin, [S.I.], v. 27, n. 2, p. 187-195, 2001.

FOSTER, K.; USHER, K.; BAKER, J. A.; GADAI, S.; ALI, S. Mental health attitudes toward mental illness in Fiji. Australian Journal of Advanced Nursing, South Melbourne, v. 25, n. 3, p. 72-9, 2010.

GIL, I. M. A.; SANTOS, J. C. P.; LOUREIRO, L. J. Estigma em estudantes de enfermagem: antes e depois do contato com pessoas com transtornos mentais. Revista Enfermagem UERJ, Rio de Janeiro, v. 24, n. 1, p. 1-7, 12 jul. 2016.

GOFFMAN, E. Estigma: notas sobre a manipulação da identidade deteriorada. Trad. Mathias Lambert. 4. ed. Rio de Janeiro: LTC, 1988.

HICKLING, F.W.; ROBERTSON-HICKLING, H.; PAISLEY, V. Desinstitucionalization and attitudes toward mental illness in Jamaica: a qualitative study. Revista Panamericana de Salud Pública, Washington DC, v. 29, n. 3, p. 169-176, mar. 2011.

LÓPEZ, M. et al. La lucha contra el estigma y la discriminación en salud mental: Una estrategia compleja basada en la información disponible. Revista de La Asociación Española de Neuropsiquiatría, Madrid, v. 28, n. 1, p. 43-83, 2008.

LUIS, M. A. V. Os rumos do cuidado em saúde mental. In: SOARES, M. H.; BUENO, S. M. V. (Org.). Saúde mental: novas perspectivas. São Paulo: Yendis, p. 55-68, 2011.

NORDT, C.; ROSSLER, W.; LAUBER, C. Attitudes of mental health professionals toward people with schizophrenia and major depression. Schizophrenia Bulletin, [Sl.], v. 32, n. 4, p. 709-714, 2006.

OMS. Organização Mundial da Saúde. Relatório mundial da saúde mental: nova concepção, nova esperança. Genebra: WHO, 2001. Disponível em: http://www.who.int/whr/. Acesso em: 05 nov. 2016.

ROMANO, A.M.M. Evolução das atitudes de alunos de enfermagem frente aos transtornos mentais, opção de escolha da profissão e preferência por áreas específicas de atuação. 2016. 104 f. Tese (Doutorado) - Escola de Enfermagem de Ribeirão Preto/USP, Ribeirão Preto, 2016. 


\section{Como referenciar este artigo}

SANTOS FERREIRA, Marcela dos. Combater o estigma associado ao sofrimento psíquico: um relato de experiência da educação profissional em saúde. Temas em Educ. e Saúde, Araraquara, v. 16, n. 1, p. 187-200, jan./jun., 2020. e-ISSN 2526-3471. DOI: https://doi.org/10.26673/tes.v16i1.13281

Submetido em: 04/02/2020

Revisões requeridas: 30/05/2020

Aprovado em: 28/06/2020

Publicado em: 19/06/2020 